\title{
Morbidity and quality of life after continent ileostomy: a comment
}

\author{
Viroj Wiwanitkit
}

Accepted: 11 September 2009 /Published online: 30 September 2009

(C) Springer-Verlag 2009

\section{Dear editor:}

I read the recent publication by Hoekstra et al. with a great interest. Hoekstra et al. reported that "The continent ileostomy remains to be a suitable alternative for the preservation of continence after a proctocolectomy, especially when an ileal pouch-anal anastomosis is not an option." No difference in resulted quality of life comparing with ileal pouch-anal anastomosis can be observed. Indeed, the surgical technique seems to have

only a few impacts on quality of life of the patients. As a concept, earlier ileostomy closure is proved for reduction the frequently occurring stoma-related morbidity and for increasing the quality of life of the patients. Nevertheless, two thirds of the cases can have early ileostomy closure. Before making a conclusion whether continent or conventional ileostomy is better, a systematic comparative assessment on cost effectiveness and cost utility is recommended.
V. Wiwanitkit $(\bowtie)$

Wiwanitkit House,

Bangkhae,

Bangkok, Thailand 10160

e-mail: wviroj@yahoo.com 